\title{
miR-19a correlates with poor prognosis of clear cell renal cell carcinoma patients via promoting cell proliferation and suppressing PTEN/SMAD4 expression
}

\author{
QIANG MA $^{1 *}$, ZHIQIANG PENG ${ }^{2 *}$, LEI WANG $^{3},{\text { YANMING } \text { LI }^{2}, \text { KAIZHEN WANG }}^{2}$, \\ JUNFANG ZHENG $^{2 * *}$, ZHIYONG LIANG ${ }^{1^{* *}}$ and TONGHUA LIU ${ }^{1 * *}$ \\ ${ }^{1}$ Department of Pathology, Peking Union Medical College Hospital, Chinese Academy of Medical Sciences and \\ Peking Union Medical College, Beijing 100730; ${ }^{2}$ Department of Biochemistry and Molecular Biology, \\ Capital Medical University, Beijing 100069; ${ }^{3}$ Department of Urology, Capital Medical University \\ Affiliated Beijing Friendship Hospital, Beijing 100050, P.R. China
}

Received August 11, 2016; Accepted October 10, 2016

DOI: 10.3892/ijo.2016.3746

\begin{abstract}
MicroRNAs (miRNAs) were reported to be involved in the development of clear cell renal cell carcinoma (ccRCC). However, the study on miRNAs in ccRCC is far from complete. The present study identified miRNAs which could act as potential novel prognostic markers for ccRCC, and analyzed its possible mechanism. We found that miR-19a correlated with poor prognosis of ccRCC patients via promoting cell proliferation and suppressing PTEN/SMAD4 expression. Both the microarray screening result and TCGA KIRC dataset analysis showed that miR-19a was significantly upregulated in ccRCC tissues, and further analysis of TCGA data revealed that the upregulated level of miR-19a was strongly associated with advanced $\mathrm{T}$ stage and poor prognosis of ccRCC patients. Consistent with clinical observations, miR-19a overexpression significantly promoted ccRCC cell proliferation in vitro. To further explore the mechanism by which miR-19a correlated with cell proliferation and poor
\end{abstract}

Correspondence to: Dr Junfang Zheng, Department of Biochemistry and Molecular Biology, Capital Medical University, No. 10 Xitoutiao, You An Men, Beijing 100069, P.R. China

E-mail: zhengjf@ccmu.edu.cn

Professor Zhiyong Liang, Department of Pathology, Peking Union Medical College Hospital, Chinese Academy of Medical Sciences and Peking Union Medical College, No. 1 Wangfujing Street, Beijing 100730, P.R. China

E-mail: liangzhiyong1220@yahoo.com

${ }^{*, * *}$ Contributed equally

Abbreviations: ccRCC, clear cell renal cell carcinoma; PTEN, phosphatase and tensin homolog deleted on chromosome ten; SMAD4, SMAD family member 4; TGF $\beta$, transforming growth factor beta

Key words: miR-19a, clear cell renal cell carcinoma, prognostic marker, PTEN, SMAD4, proliferation prognosis of ccRCC, we performed gene set enrichment analysis (GSEA) for target genes of miR-19a in ccRCC patients. Result indicated that the key target genes of miR-19a included SMAD4 and PTEN. In ccRCC tissues, expression levels of SMAD4 and PTEN were negatively correlated with expression level of miR-19a, revealing that miR-19a suppressed the expression of SMAD4 and PTEN in ccRCC patients. miR-19a overexpression significantly suppressed the expression of SMAD4 and PTEN in vitro, further verifying that SMAD4 and PTEN were the target genes of miR-19a in ccRCC cells. Our results elucidated the tumor promoting role of miR-19a and established miR-19a as a potential novel prognostic marker for ccRCC.

\section{Introduction}

Renal cell carcinoma (RCC) is one of the most common and lethal renal types of cancer, reducing the life quality of patients and presenting as an important socioeconomic problem. Incidence and mortality rates of RCC have increased in recent years (1). Clear cell RCC (ccRCC) is the most common subtype (70-80\%) of RCC (2). Although many genetic and epigenetic changes were found to correlate with ccRCC, the pathogenesis remains poorly understood.

MicroRNAs (miRNAs), emerging as endogenous triggers of the RNA interference pathway, were abnormally expressed in multiple types of cancer playing a significant role in the carcinogenesis or prognosis prediction $(3,4)$. miRNAs exerted their functions by binding the 3' untranslated regions (UTRs) of target mRNAs to suppress target gene expression and the control of various biological processes, such as cell proliferation, apoptosis, differentiation and migration (5-8). Hence, deregulated miRNAs have triggered considerable interest in using them as prognostic markers and molecular therapeutic targets for cancer (9).

During ccRCC occurence and progression, the abnormal expression of miRNAs was also found to exert important functions (10). miR-21, miR-122-5p and miR-149 were upregulated in ccRCC tissues $(11,12)$. In contrast, miR-10b and miR-27b, 
were downregulated in ccRCC tissues $(13,14)$. These miRNAs were also reported to be involved in the development of ccRCC. However, miRNAs influencing ccRCC occurrence and progression need further investigation. There is no miRNA marker for use in the clinic. Thus, there is still a growing need for prognostic markers and therapeutic targets to improve the outcomes of ccRCC patients.

In the present study, we found that miR-19a was significantly upregulated in ccRCC tissues. High level of miR-19a was correlated with the progression and poor prognosis of ccRCC and promoted ccRCC cell proliferation. miR-19a exerted these functions by regulating multiple types of cancerrelated pathways and mainly targeting SMAD4 and PTEN. These results helps to elucidate the pathogenesis and predict prognosis of ccRCC.

\section{Materials and methods}

Patient tissues. Surgical specimens of $18 \mathrm{ccRCC}$ cases and adjacent normal renal tissues were collected between January 2013 and December 2014 in the Capital Medical University Affiliated Beijing Friendship Hospital (Beijing, China). The study was approved by the Research Ethics Boards of both Capital Medical University and Beijing Friendship Hospital. All subjects included in the protocol signed a declaration of informed consent. The tumor specimens were classified according to the 2010 American Joint Committee on Cancer (AJCC) staging system.

RNA isolation. Total RNA was extracted from ccRCC and normal renal tissues using the TRIzol reagent (Invitrogen, Carlsbad, CA, USA) according to the manufacturer's protocol. RNA quantity and purity were assessed using NanoDrop ND-1000 (Thermo Fisher Scientific, Waltham, MA, USA). The criteria for absorbance ratios are established at A260/ A280 $\geq 1.8$ and A260/A230 $\geq 1.5$ indicating acceptable RNA purity. RNA integrity number (RIN) values were ascertained using Agilent RNA 6000 Nano assay (Agilent Technologies, Santa Clara, CA, USA). The criteria for RIN value is established at $\geq 5$ indicating acceptable RNA integrity. Genomic DNA contamination was evaluated by gel electrophoresis. The RNA samples were stored at $-80^{\circ} \mathrm{C}$ until analysis.

Microarray analysis. miRNA microarray analysis was performed by the Human miRNA OneArray ${ }^{\circledR}$ v6 (Phalanx Biotech Group, Inc., Hsinchu, Taiwan). It contains triplicate 2539 unique miRNA probes from human (miRBase release v20) each printed in technical triplicate, and 114 experimental control probes. Small RNA was pre-enriched by Nanoseplook (Pall Corp., Port Washington, NY, USA) from $2.0 \mu \mathrm{g}$ total RNA samples and labeled with miRNA ULS ${ }^{\mathrm{TM}}$ labeling kit (Kreatech Diagnostics, Amsterdam, The Netherlands). Labeled miRNA targets were hybridized to the human miRNA OneArray ${ }^{\circledR}$ v6 with OneArray ${ }^{\circledR}$ hybridization system. After $16 \mathrm{~h}$ of hybridization at $37^{\circ} \mathrm{C}$, non-specific binding targets were washed away by three different washing steps (wash I $37^{\circ} \mathrm{C}$ for $5 \mathrm{~min}$; wash II $37^{\circ} \mathrm{C}$ for $5 \mathrm{~min}, 25^{\circ} \mathrm{C}$ for $5 \mathrm{~min}$; wash III rinse 20 times), and the slides were dried by centrifugation and scanned by an Axon 4000B scanner (Molecular Devices, Sunnyvale, CA, USA). The Cy5 fluorescent intensities of each probe were analyzed by GenePix 4.1 software (Molecular Devices). The raw intensity of each probe was processed by $\mathrm{R}$ program. Probes that passed the criteria were normalized by $75 \%$ median scaling normalization method. Normalized spot intensities were transformed to miRNA expression $\log _{2}$ ratios between tumor tissues and adjacent normal tissues from ccRCC patients.

The Cancer Genome Atlas (TCGA) data. The TCGA data on miRNA, mRNA (RNASeq v2) and protein (RPPA) expression levels in ccRCC patients were obtained from https://www. synapse.org/. The miR-19a level, SMAD4 mRNA level, PTEN protein level and whole transcriptome data were used in the present study. Clinical data were downloaded from cBioPortal database (www.cbioportal.org).

Gene set enrichment analysis. The association between expression level of miR-19a and biological processes/ microRNA target gene set was analyzed using Gene set enrichment analysis (GSEA v2.2, http://www.broad.mit.edu/ gsea/). GSEA calculates a gene set enrichment score (ES) that estimates whether genes from pre-defined gene set (obtained from the Molecular Signatures Database, MSigDB, regulation of cell proliferation, http://software.broadinstitute.org; miR-19a target gene set, http://mirtarbase.mbc.nctu.edu.tw/) are enriched among the highest- (or lowest-) ranked genes or distributed randomly. Default settings were used. Thresholds for significance were determined by permutation analysis (1000 permutations). False discovery rate (FDR) was calculated. A gene set is considered significantly enriched when the FDR score is $<0.05$.

Cell culture and miRNA transfection. Human ccRCC cell line 769-P was purchased from the Cell Resource Center of Beijing Xiehe (Beijing, China) and cultivated in an incubator at $37^{\circ} \mathrm{C}$ with $5 \% \mathrm{CO}_{2} .769-\mathrm{P}$ was maintained in Roswell Park Memorial Institute (RPMI)-1640 medium (Gibco, Waltham, MA, USA) supplemented with $10 \%$ fetal bovine serum (FBS; HyClone Laboratories, Inc., Logan, UT, USA) as well as penicillin (100 U/ml; Thermo Fisher Scientific).

miR-19a mimics and its negative control (NC) sequences were designed and synthesized by Sangon Biotech, Co., Ltd. (Shanghai, China). Lipofectamine 2000 (Invitrogen) was used for miRNA transfection according to the manufacturer's protocol. After $48 \mathrm{~h}$ of transfection, cells were used for the proliferation and western blot experiments.

CCK-8 assay. Cells transfected with miR-19a mimics or NC sequence were plated at a density of $5 \times 10^{3}$ cells/well onto 96-well plates at $37^{\circ} \mathrm{C}$ in an incubator with $5 \% \mathrm{CO}_{2}$. Cell proliferation was then assessed every $24 \mathrm{~h}$ using Cell Counting kit-8 (CCK-8; Sigma-Aldrich, St. Louis, MO, USA) according to standard protocol. For each sample at each time-point, 6 wells were analyzed. The experiment was repeated three times.

Gene Ontology and KEGG pathway analyses. miRPath v3.0 was used to map the Gene Ontology (GO) of 39 differentially expressed miRNAs and unifying functional KEGG (Kyoto Encyclopedia of Genes and Genomes) pathways of miR-19a in DIANA Tools database (http://snf-515788.vm.okeanos.grnet. 
$\mathrm{gr} /)$. To explore the functional annotation enrichment of the leading edge target genes of miR-19a in ccRCC, the GO and KEGG analyses were conducted using the WebGestalt (the Database for functional genomic, proteomic and large-scale genetic studies) online analysis tool (http://bioinfo.vanderbilt. edu/webgestalt/option.php) (15).

Protein-protein interaction (PPI) network construction. Search Tool for the Retrieval of Interacting Genes/Proteins (STRING; Search Tool for the Retrieval of Interacting Genes, http://string-db.org/) is a database of known and predicted protein interactions that may aid in the comprehensive description of cellular mechanisms and functions. The PPI network of the selected leading edge target genes of miR-19a in ccRCC was constructed using the STRING database.

IHC data. The IHC-based protein expression data including high-resolution images were viewed and downloaded from the Human Protein Atlas web portal (www.proteinatlas.org).

Western blotting. Cells were collected and lysed in lysis buffer (Beijing CoWin Biotech, Co., Ltd., Beijing, China) in the presence of protease inhibitors for $30 \mathrm{~min}$ to extract total protein from cells transfected with miR-19a mimics or negative control, and protein levels were quantified using bicinchoninic acid assays (Beijing CoWin Biotech). Subsequently, $30 \mu \mathrm{g}$ protein from each sample was loaded onto $10 \%$ sodium dodecyl sulfate (SDS) polyacrylamide gels and subjected to SDS-polyacrylamide gel electrophoresis (PAGE; Beijing CoWin Biotech). Protein was then transferred to nitrocellulose membranes (Sigma-Aldrich), which were blocked with bovine serum albumin blocking buffer (Invitrogen) for $1 \mathrm{~h}$. Membranes were then incubated with primary antibodies targeting SMAD4 (1:2,000 dilution, cat. no. ab40759; Abcam), PTEN (1:1,000 dilution, cat. no. ab32199; Abcam), or GAPDH (1:5000 dilution, cat. no. ab70699; Abcam) overnight at $4^{\circ} \mathrm{C}$, followed by incubation with goat anti-rabbit horseradish peroxidase (HRP)-conjugated secondary antibody (1:3,000 dilution, cat. no. CW0103; Beijing CoWin Biotech) for $1 \mathrm{~h}$ at room temperature. Detection was facilitated using an enhanced chemiluminescence kit and images were analyzed using ImageJ software (version 1.62; National Institute of Health, Bethesda, MD, USA).

Statistical analysis. Statistical analyses were performed using SPSS 19.0 (SPSS, Inc., Chicago, IL, USA) and Graphpad Prism 5 (Graphpad Software, Inc., San Diego, CA, USA). miRNA microarray data and TCGA data for paired samples were analyzed by paired sample t-test. TCGA and IHC data for unpaired samples were analyzed by independent samples t-test. The relationship between miR-19a expression level and clinical stages was analyzed by one-way ANOVA. The effect of miR-19a on the constituent ratio of clinical stages was explored by Pearson's Chi-square test. The log-rank test for the generated Kaplan-Meier curve was conducted to evaluate the association between the expression level of miR-19a and the survival rate. Overall survival (OS) was defined as the time between the first surgery for primary ccRCC and death for any reason. Disease-free survival (DFS) was defined as the time between the first surgical resection and disease recurrence.
Table I. Dysregulated miRNAs related to ccRCC cell proliferation.

\begin{tabular}{|c|c|c|c|}
\hline No. & Name & $\begin{array}{c}\log _{2} \\
\text { (T/N fold change) }\end{array}$ & P-value \\
\hline 1 & miR-21-5p & 5.59757949 & 0.000565035 \\
\hline 2 & miR-143-3p & 3.956144756 & 0.000384443 \\
\hline 3 & miR-126-3p & 3.884555682 & 0.000405514 \\
\hline 4 & miR-92a-3p & 3.744235089 & 0.003313711 \\
\hline 5 & miR-16-5p & 3.613193175 & 0.00066429 \\
\hline 6 & $\operatorname{miR}-23 a-3 p$ & 3.601969832 & 0.004209703 \\
\hline 7 & miR-24-3p & 3.549553664 & 0.000561719 \\
\hline 8 & miR-106b-5p & 3.176934445 & 0.00000023 \\
\hline 9 & $\operatorname{miR}-27 a-3 p$ & 3.101813543 & 0.003764223 \\
\hline 10 & $\operatorname{miR}-22-3 p$ & 3.040650864 & 0.001954592 \\
\hline 11 & miR-19b-3p & 2.743074269 & 0.001962923 \\
\hline 12 & miR-10b-5p & 2.648244991 & 0.003323708 \\
\hline 13 & $\operatorname{miR}-185-3 p$ & 2.46487356 & 0.000747848 \\
\hline 14 & miR-15a-5p & 2.403516915 & 0.003705642 \\
\hline 15 & $\operatorname{miR}-146 a-5 p$ & 2.259132968 & 0.00096887 \\
\hline 16 & $\operatorname{miR}-25-3 p$ & 2.117041931 & 0.002269416 \\
\hline 17 & miR-181b-5p & 2.089754653 & 0.007906868 \\
\hline 18 & miR-106a-5p & 2.045900851 & 0.0000192 \\
\hline 19 & miR-20a-5p & 2.032254678 & 0.000533588 \\
\hline 20 & miR-17-5p & 1.923456726 & 0.000000293 \\
\hline 21 & miR-19a-3p & 1.913744267 & 0.00296129 \\
\hline 22 & $\operatorname{miR}-221-3 p$ & 1.887913841 & 0.00000315 \\
\hline 23 & miR-222-3p & 1.820039677 & 0.000792998 \\
\hline
\end{tabular}

Univariate and multivariate Cox proportional hazard regression analyses were used to estimate the prognostic significance of miR-19a in ccRCC patients. Growth curves were analyzed using a repeated-measures ANOVA followed by Bonferroni post hoc analysis. The correlation of miR-19a and target gene expression levels were analyzed by Pearson correlation analysis. $\mathrm{P}<0.05$ was considered to indicate a statistically significant difference.

\section{Results}

Expression level of miR-19a is upregulated in ccRCC tissues. To identify the novel differentially expressed miRNAs related with ccRCC occurrence and development, miRNA microarray and TCGA_KIRC miRNA dataset were used in the present study. Eighteen pairs of ccRCC tissues and adjacent normal renal tissues were compared by miRNA microarray, 152 differentially expressed miRNAs $(\mathrm{P}<0.01 ; \mathrm{FC}>3$ and $\mathrm{FC}<0.33)$ were identified. Of which, 39 miRNAs are also consistently differentially expressed $(\mathrm{P}<0.05)$ in TCGA_KIRC miRNA seq v2 dataset. Cell proliferation is an important process of cancer development and progression (16) and proliferation related proteins predicted the poor outcome of cancer patients (17). To identify differentially expressed miRNAs correlated with cell proliferation, we searched GO annotation of 39 miRNAs. Among 39 dysregulated miRNAs, 23 miRNAs were annotated with cell proliferation. Notably, these 23 miRNAs were all upregulated in ccRCC tissues. miR-19a was one of 


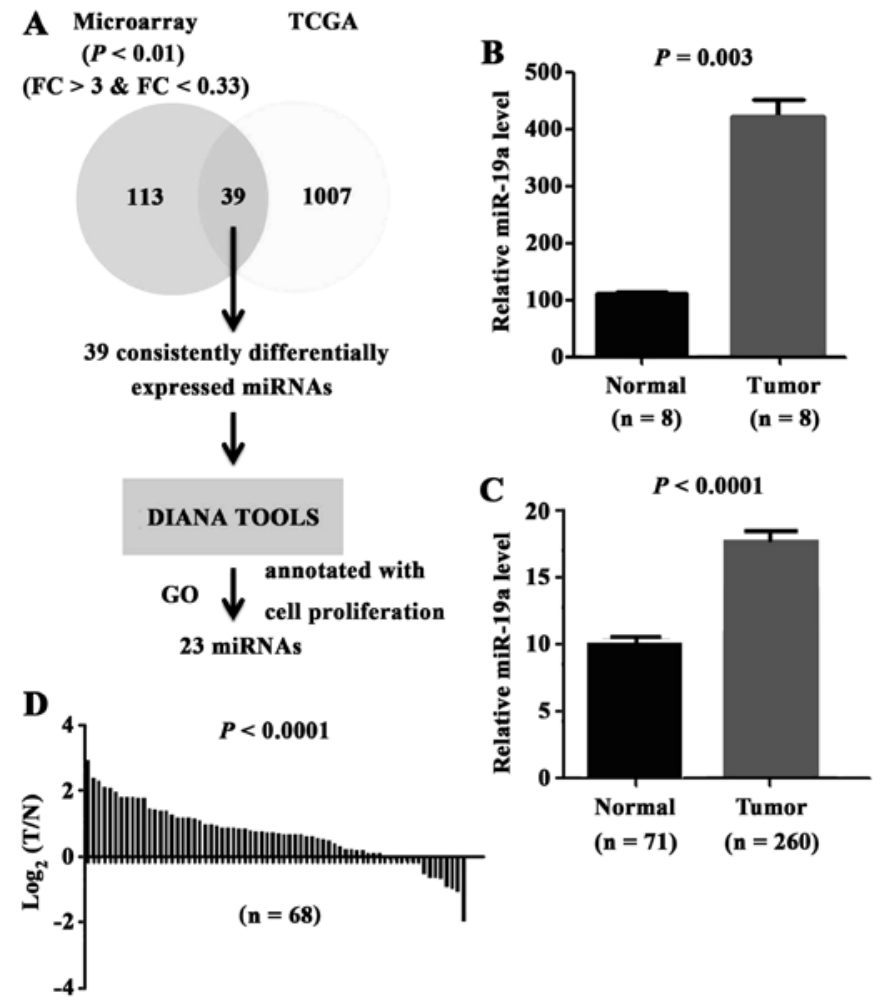

Figure 1. Expression level of miR-19a in ccRCC tissues is upregulated. (A) Flow chart of screening for differentially expressed miRNAs in ccRCC. Thirty-nine miRNAs were consistently differentially expressed in microarray and TCGA KIRC dataset. Of them, 23 miRNAs were annotated with cell proliferation. (B) The upregulated expression of miR-19a in ccRCC tissues compared with adjacent normal tissues by miRNA microarray. (C) The upregulated expression of miR-19a in ccRCC tissues compared with normal tissues from TCGA KIRC dataset. (D) miR-19a expression level was upregulated in ccRCC tissues compared with matched adjacent normal tissues in TCGA KIRC dataset.

rarely reported miRNAs in ccRCC and attracted our interest (Fig. 1A-C and Table I).

To further validate the abnormally upregulated expression level of miR-19a, we compared 68 pairs of ccRCC tissue specimens from TCGA database. Consistent with the results from unpaired samples, miR-19a was significantly upregulated in tumor tissue specimens compared with matched normal controls (Fig. 1D; $\mathrm{P}<0.0001$ ).

Upregulated miR-19a is associated with advanced stage and poor prognosis of $c c R C C$. To elucidate the role of miR-19a in ccRCC progression, the expression level of miR-19a in different stages of ccRCC patients was analyzed. The results revealed that with the increase of ccRCC stage, miR-19a expression level increased accordingly $(\mathrm{P}<0.0001$; Fig. $2 \mathrm{~A})$, indicating the role of upregulated miR-19a in ccRCC progression and prognosis prediction. To further observe the role of miR-19a expression in ccRCC progression, constituent ratio analysis was performed in an expanded set of 236 primary ccRCC tissues and 71 normal samples. In the normal renal tissues, only 10/71 (14\%) cases exhibited high expression level for miR-19a (>median). However, in I/II stage and III/IV stage of ccRCC tissues, $81 / 144(56 \%)$ of cases and 62/92 (67\%) of cases showed high expression level for miR-19a, respectively. The percentage with high level of miR-19a was drastically different between normal
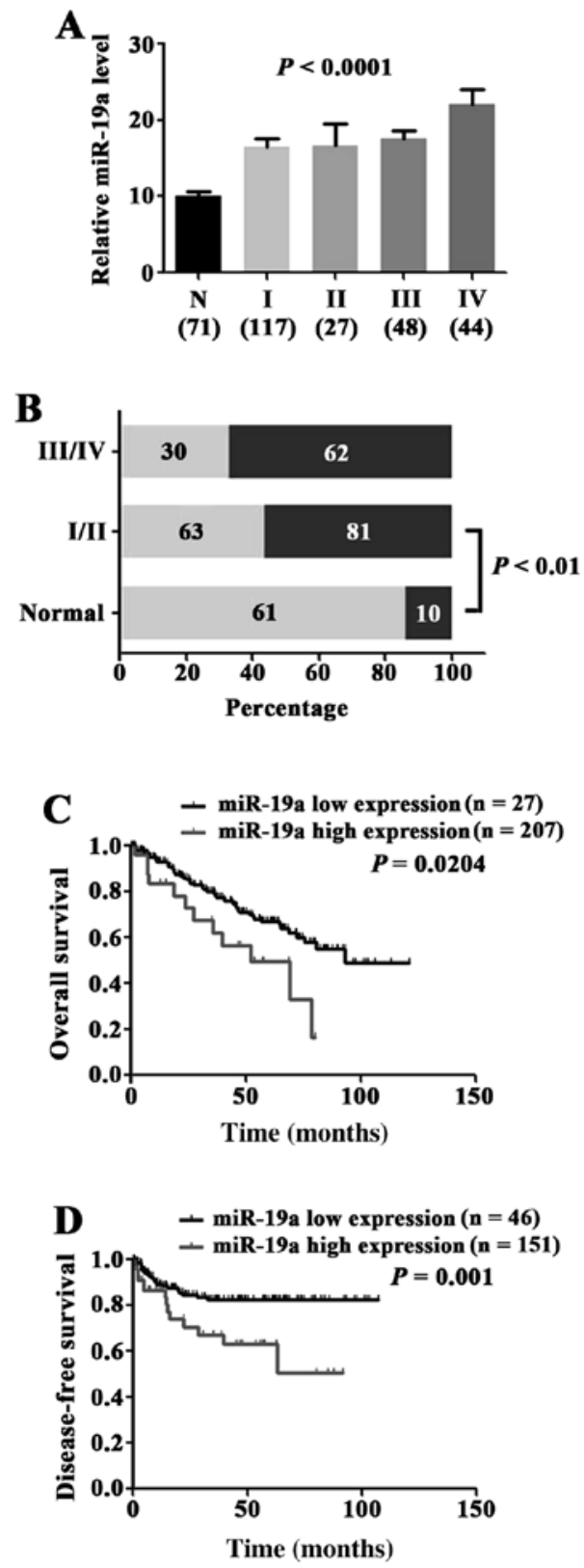

Figure 2. The correlation between expression level of miR-19a and progression/prognosis of ccRCC patients. (A) Correlation analysis was performed between expression level of miR-19a and clinical AJCC stages. miR-19a was correspondingly increased with the increase of clinical AJCC stage of ccRCC. (B) Constituent ratio with high level of miR-19a was drastically different between normal samples and I/II or III/IV stage of ccRCC tissues. (C) Kaplan-Meier curve for OS of patients with high $(n=207)$ and low $(n=27)$ miR-19a expression level. Results revealed that high miR-19a expression level was correlated with shorter OS for ccRCC patients. (D) miR-19a high expression group $(n=151)$ had shorter DFS than miR-19a low expression group $(n=46)$.

samples and ccRCC I/II or III/IV stage ccRCC tissues $(\mathrm{P}<0.01)$, suggesting that miR-19a was associated with ccRCC progression and might have prognostic significance for ccRCC patients (Fig. 2B). Thus, we further investigated the prognostic role of miR-19a expression level as a prognostic marker. The patients were divided into miR-19a high expression and miR-19a low expression groups. Kaplan-Meier curve showed that miR-19a high expression group had shorter OS and DFS than miR-19a low expression group in terms of survival duration $(\mathrm{P}<0.05$; 
Table II. Univariate and multivariate analyses of miR-19a expression and disease-free survival of patients.

\begin{tabular}{|c|c|c|c|c|c|c|}
\hline \multirow[b]{2}{*}{ Variables } & \multicolumn{3}{|c|}{ Univariate analysis } & \multicolumn{3}{|c|}{ Multivariate analysis ${ }^{\mathrm{a}}$} \\
\hline & $\mathrm{HR}^{\mathrm{b}}$ & $95 \% \mathrm{CI}^{\mathrm{c}}$ & $\mathrm{P}$-value & $\mathrm{HR}^{\mathrm{b}}$ & $95 \% \mathrm{CI}^{\mathrm{c}}$ & $\mathrm{P}$-value \\
\hline
\end{tabular}

Disease-free survival $(\mathrm{n}=197)$

Age (years)

$$
\begin{aligned}
& \geq 60(n=89) \\
& <60(n=108)
\end{aligned}
$$

$0.994-1.033$

0.625

Gender

Female $(\mathrm{n}=62)$

Male $(n=135)$

0.809

$0.379-1.728$

0.584

Stage

$$
\begin{aligned}
& \text { III/IV }(n=71) \\
& \text { I/II }(n=126)
\end{aligned}
$$

3.962-20.87

0.000

9.181

3.993-21.11

0.000

miR-19a

High $(\mathrm{n}=151)$

Low $(n=46)$

${ }^{a}$ Multivariate models were adjusted for stage classification, age and gender. ${ }^{b}$ Hazard ratio, estimated from Cox proportional hazard regression model. 'Confidence interval of the estimated HR.
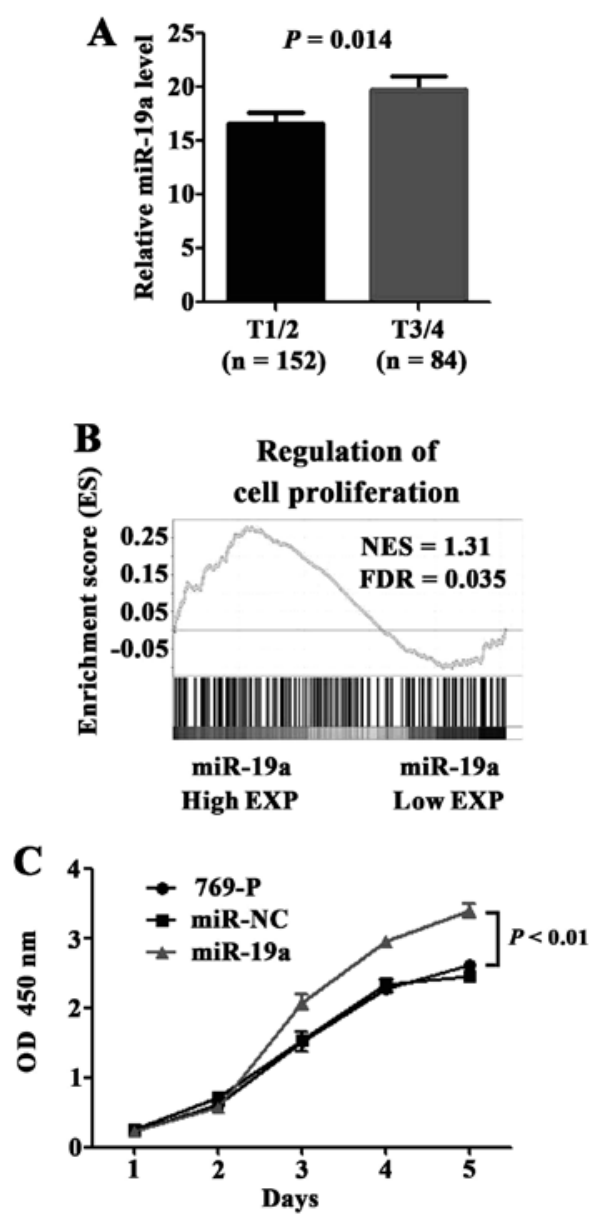

Figure 3. miR-19a promotes ccRCC cell proliferation. (A) The expression level of miR-19a in T3/4 stage ccRCC specimen was significantly higher than in T1/2 stage. (B) miR-19a high expression was positively correlated with cell proliferation in ccRCC patients. (C) miR-19a significantly promoted the proliferation of 769-P cells by $>50 \%$ at all time-points following $48 \mathrm{~h}$ of incubation.
Fig. 2C and D), particularly in DFS. The result suggests that upregulated miR-19a may be a novel prognostic marker for ccRCC patients. Univariate and multivariate Cox regression analyses further revealed miR-19a could be an independent prognostic marker for ccRCC patients (Table II).

miR-19a promotes cell proliferation of $c c R C C$. To specify which of the T, N and M stages was significantly related with miR-19a expression level, we next, respectively, investigated the relationship of miR-19a expression level with T, $\mathrm{N}$ and $\mathrm{M}$ stage. miR-19a expression level was most significantly related with $\mathrm{T}$ stage $(\mathrm{P}<0.05$; Fig. $3 \mathrm{~A})$. $\mathrm{T}$ stage is closely related to tumor size and cell proliferation, revealing that miR-19a was significantly related with cell proliferation of ccRCC patients. GSEA was used to further verify the relationship of miR-19a expression level with cell proliferation in ccRCC patients. The ccRCC patients from TCGA dataset were divided into high and low miR-19a expression groups, and correlation between miR-19a expression level and cell proliferation gene set was analyzed. As shown in Fig. 3B, the gene set of cell proliferation was highly enriched in the miR-19a high expression group (FDR <0.05). The data suggest miR-19a expression level positively correlates with cell proliferation. Next, the ability of miR-19a in modulating ccRCC cell proliferation was analyzed in vitro. The results indicated that miR-19a overexpression significantly promoted the proliferation of 769-P cells by $>50 \%$ at all time-points following $48 \mathrm{~h}$ of incubation, as compared with control cells $(\mathrm{P}<0.01)$. Moreover, no significant difference was observed between untransfected cells and negative control sequence-transfected cells (Fig. 3C). These data suggest that miR-19a promotes ccRCC cell proliferation.

miR-19a is involved in multiple types of cancer related pathways. miRNAs were reported to promote cancer occurence and 

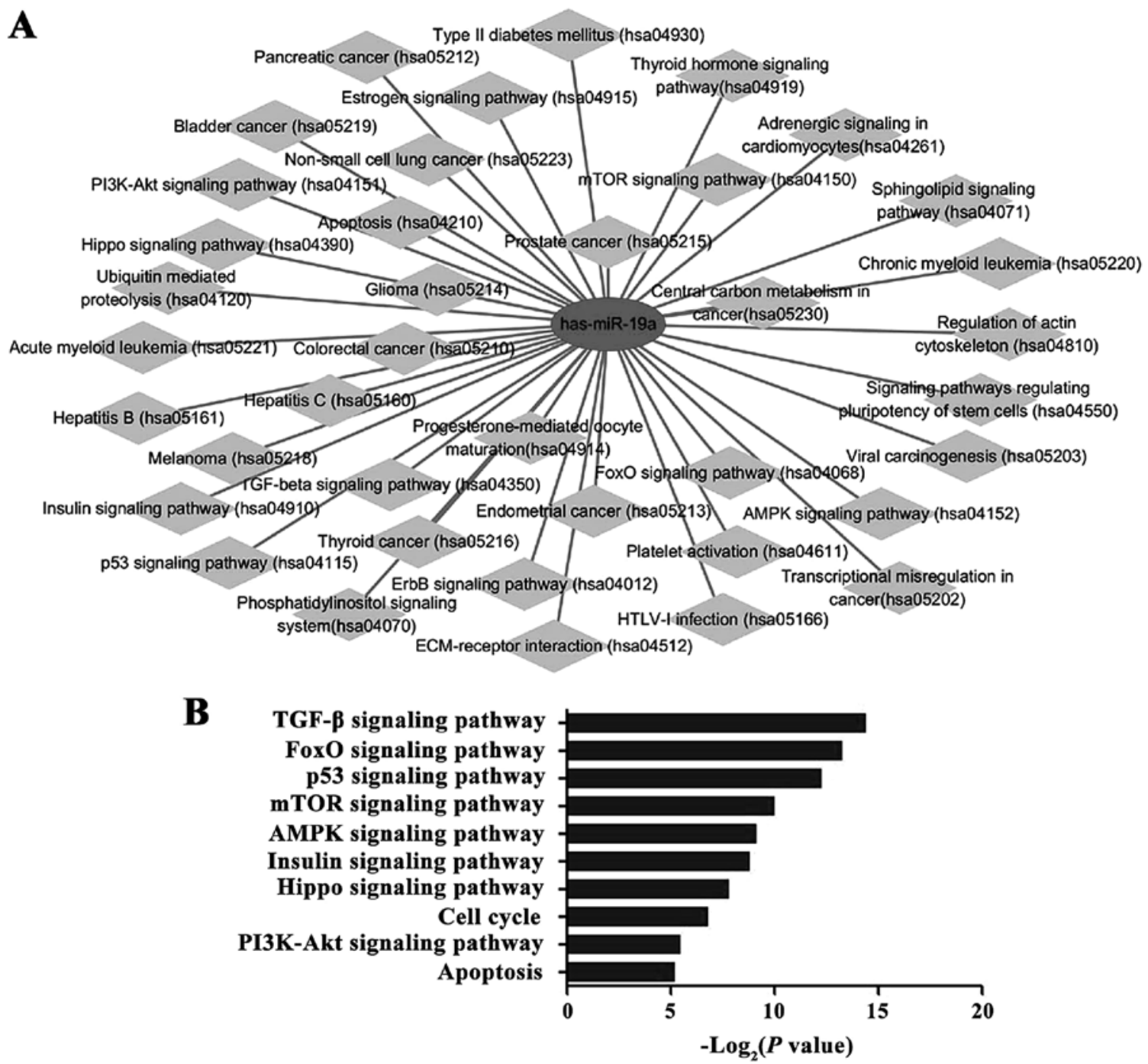

Figure 4. miR-19a is involved in multiple types of cancer-related pathways. (A) miR-19a was involved in 49 significant KEGG pathways. (B) The miR-19a relevant main signaling pathways.

progression through activating related signaling pathways (18). To better understand the underlying molecular mechanism by which miR-19a functions, we investigated miR-19a relevant KEGG pathways. Through the miRNA-targeted pathway union analysis, we found that miR-19a was involved in 49 significant KEGG pathways $(\mathrm{P}<0.05)$. These pathways mainly included multiple types of cancer related signaling pathways, especially the TGF- $\beta$ signaling pathway, mTOR signaling pathway and PI3K-Akt signaling pathway (Fig. 4).

PTEN and SMAD4 are key target genes of miR-19a in $c c R C C$. miRNAs exerted important functions in various biological processes via directly suppressing the expression of their target genes (19). However, for the same miRNA, target genes and involved pathways are different in different tissues (20). To find out the key and the specific target genes of miR-19a in ccRCC patients, we chose 28 target genes of miR-19a which as identified in the miRTarBase dataset and whose expression level value was listed in the TCGA KIRC dataset, investigated their correlation with miR-19a in ccRCC by GSEA. The results revealed that 19 target genes were significantly enriched in miR-19a low expression group, suggesting that they are the main target genes of miR-19a in ccRCC patients (Fig. 5A and Table III). These genes included SMAD4, PTEN, BCL2L11, SUZ12, PSAP, THBS1, KAT2B, VPS4B and BMPR2. Especially, SMAD4 and PTEN were the high ranked target genes for miR-19a. We further performed GO and KEGG pathway analysis for these target genes. We noted that the 19 genes were especially enriched in functions of metabolic process, biological regulation, macromolecular complex and protein binding (Fig. 5B). Another functional analysis of above target genes by KEGG annotation revealed that some signal transduction pathways, such as transforming growth factor (TGF)- $\beta$ signaling pathway, pathway in cancer and p53 signaling pathway were significantly regulated by miR-19a (Fig. 5C). In addition, KEGG pathway analysis results also revealed that SMAD4 and PTEN were involved in many cell proliferation-related signaling pathways (data not shown). Since macromolecular complex and protein binding are the important molecular functions for target genes, STRING was used to analyze the interaction among the 19 target genes of miR-19a. SMAD4 and PTEN were found to play important 


\section{B1 Biological Process}
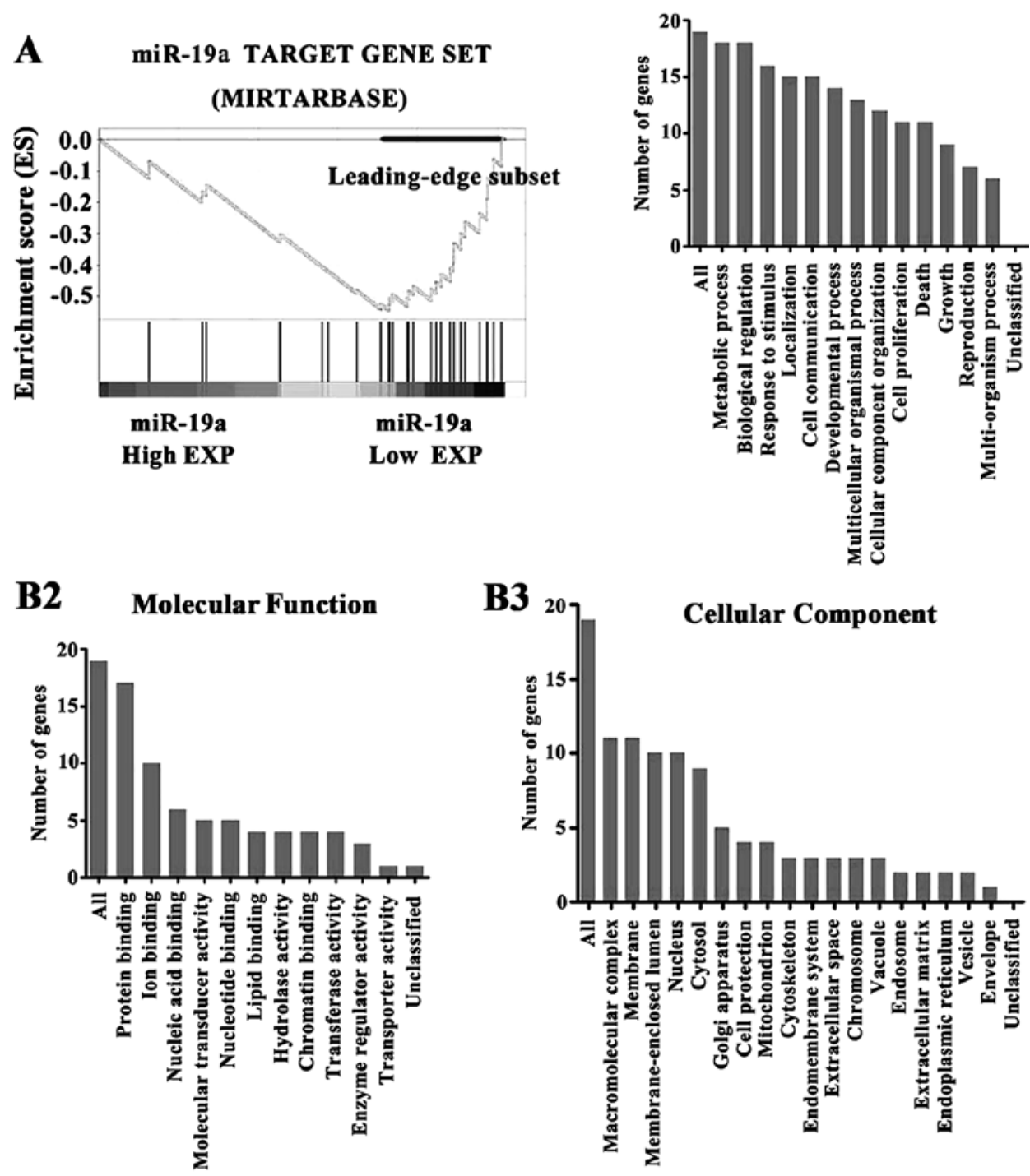

C

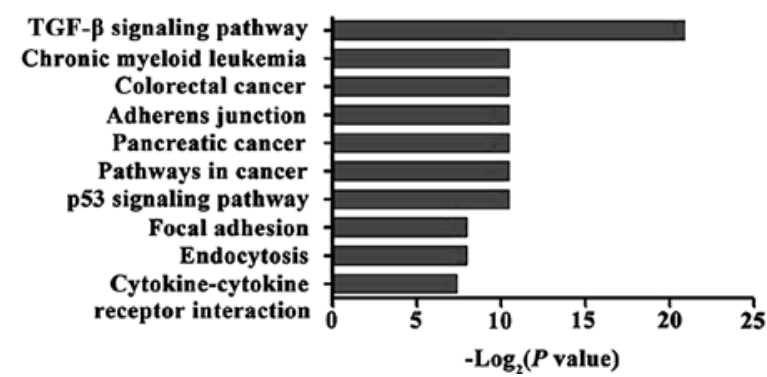

Figure 5. GSEA, GO, KEGG and PPI analyses for the main target genes of miR-19a in ccRCC. (A) Nineteen genes were significantly enriched in miR-19a low expression group; they are the main target genes of miR-19a in ccRCC. The curve was the enrichment score of these genes by GSEA. The black horizontal line (on the top) denotes the leading edge subset of the main target genes for miR-19a in ccRCC patients. (B) GO analysis of the main target genes according to biological process, molecular function and cellular component. (C) KEGG pathway analysis of the main target genes.

roles in this network (Fig. 5D). In summary, data suggest that miR-19a promotes ccRCC occurrence, progression and poor prognosis mainly via suppressing SMAD4 and PTEN expression.

SMAD4 and PTEN expression levels are downregulated by miR-19a in ccRCC. SMAD4 and PTEN are well-known tumor suppressors in multiple types of tumors $(21,22)$. The abnormal downregulation of PTEN and SMAD4 protein plays an important role in the malignant transformation of tumors $(23,24)$. In ccRCC tissues $(\mathrm{n}=11)$, SMAD4 and PTEN protein expression levels were significantly downregulated (Fig. 6A, $\mathrm{P}=0.0023$; $\mathrm{P}<0.0001$, respectively) compared with normal renal tissues from the Human Protein Atlas Database $(n=3)$. To further 


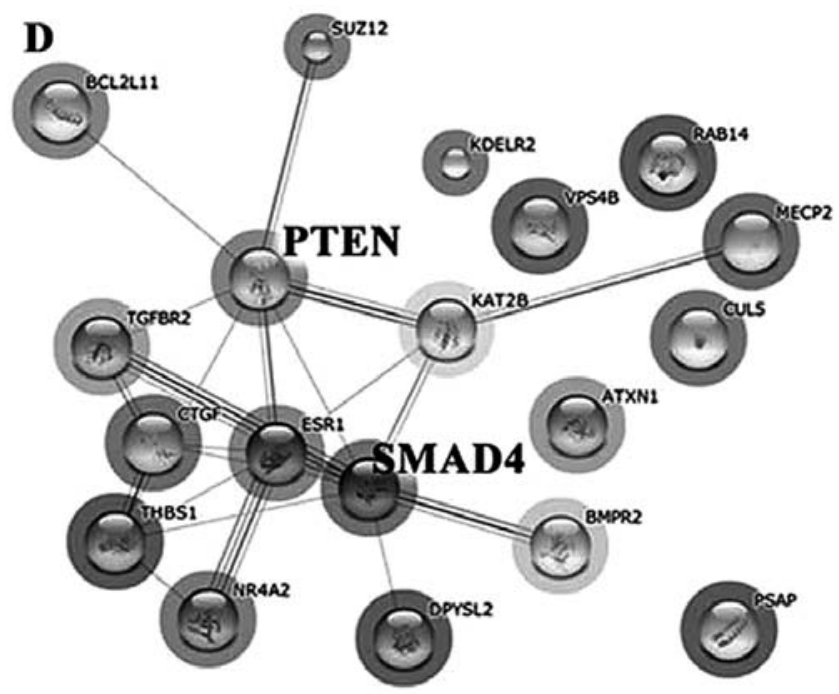

Figure 5. Continued. (D) PPI analysis of the main target genes. Thirteen of 19 target proteins showed the interaction. PTEN and SMAD4 were the key node proteins for the interaction network.

Table III. miR-19a target genes ranked by GSEA.

\begin{tabular}{|c|c|c|c|c|c|}
\hline No. & Gene symbol & Rank in gene list & Rank metric score & Running ES & Core enrichment \\
\hline 1 & ARIH2 & 1560 & 0.211 & -0.0694 & No \\
\hline 2 & RAB13 & 3222 & 0.135 & -0.1662 & No \\
\hline 3 & KIT & 3372 & 0.13 & -0.145 & No \\
\hline 4 & ABCA1 & 5676 & 0.048 & -0.3143 & No \\
\hline 5 & SOCS1 & 5689 & 0.048 & -0.3032 & No \\
\hline 6 & CCND1 & 7000 & 0.004 & -0.4055 & No \\
\hline 7 & RNF4 & 7179 & -0.002 & -0.4189 & No \\
\hline 8 & TLR2 & 8084 & -0.037 & -0.4808 & No \\
\hline 9 & PRMT5 & 8830 & -0.065 & -0.523 & No \\
\hline 10 & MECP2 & 9086 & -0.075 & -0.5242 & Yes \\
\hline 11 & KDELR2 & 9103 & -0.076 & -0.5064 & Yes \\
\hline 12 & NR4A2 & 9203 & -0.079 & -0.4941 & Yes \\
\hline 13 & CUL5 & 9659 & -0.099 & -0.505 & Yes \\
\hline 14 & ATXN1 & 9717 & -0.101 & -0.4839 & Yes \\
\hline 15 & CTGF & 9849 & -0.106 & -0.4674 & Yes \\
\hline 16 & RAB14 & 10419 & -0.135 & -0.4782 & Yes \\
\hline 17 & TGFBR2 & 10566 & -0.141 & -0.4542 & Yes \\
\hline 18 & ESR1 & 10732 & -0.151 & -0.429 & Yes \\
\hline 19 & DPYSL2 & 11026 & -0.167 & -0.4099 & Yes \\
\hline 20 & SMAD4 & 11120 & -0.172 & -0.3737 & Yes \\
\hline 21 & BCL2L11 & 11129 & -0.173 & -0.3307 & Yes \\
\hline 22 & SUZ12 & 11366 & -0.189 & -0.3015 & Yes \\
\hline 23 & PSAP & 11491 & -0.197 & -0.2614 & Yes \\
\hline 24 & THBS1 & 11947 & -0.236 & -0.2375 & Yes \\
\hline 25 & KAT2B & 12174 & -0.261 & -0.1893 & Yes \\
\hline 26 & VPS4B & 12188 & -0.262 & -0.1241 & Yes \\
\hline 27 & PTEN & 12365 & -0.292 & -0.0641 & Yes \\
\hline 28 & BMPR2 & 12636 & -0.364 & 0.0068 & Yes \\
\hline
\end{tabular}

verify the expression levels, SMAD4 and PTEN were regulated by miR-19a from clinical aspect, the correlation analyses between expression levels of SMAD4/PTEN and miR-19a were done in ccRCC tissues. The results showed that the expression 

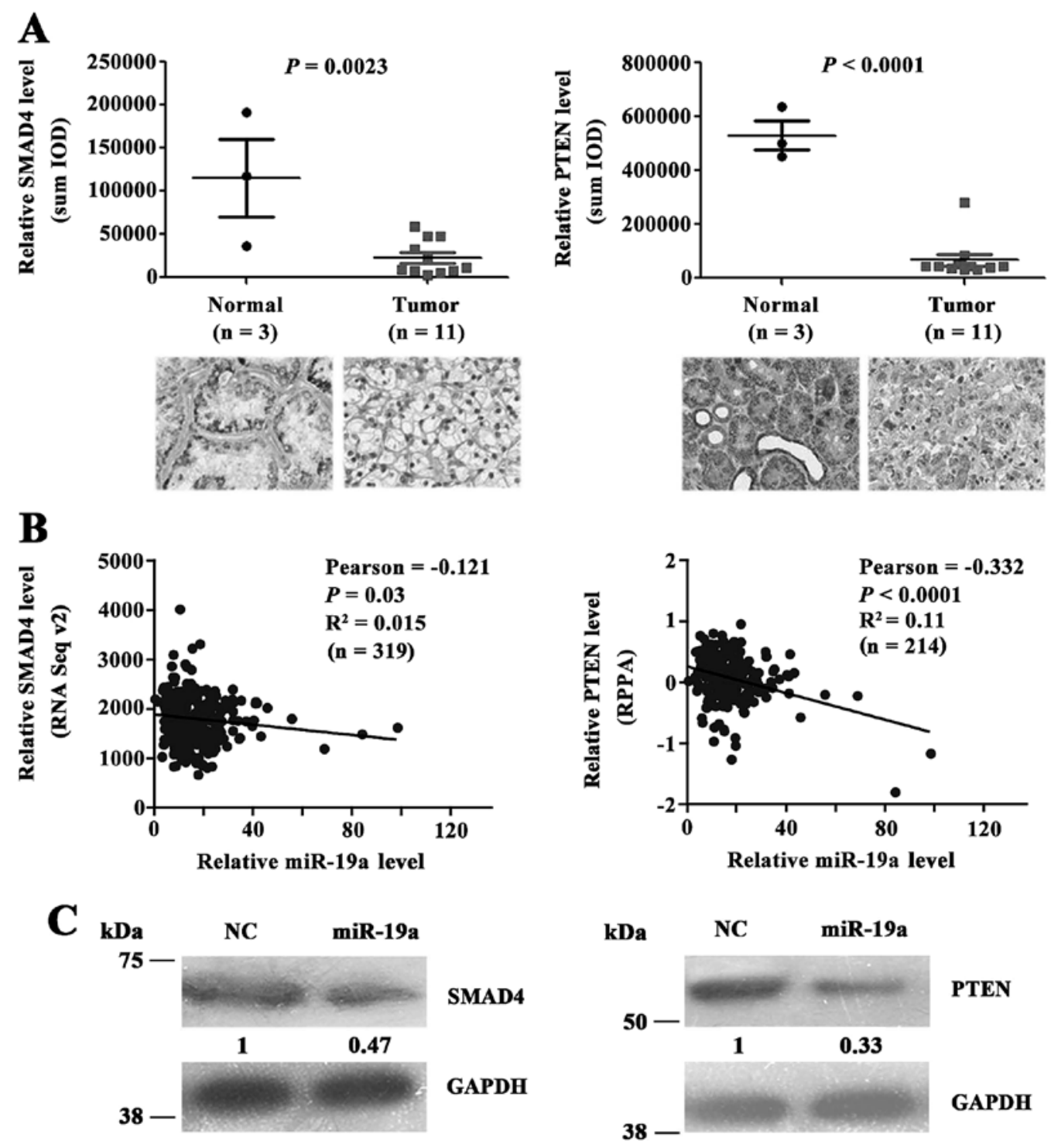

Figure 6. miR-19a suppresses the expression of SMAD4 and PTEN in ccRCC. (A) The Human Protein Atlas database results revealed that the expression level of SMAD4 and PTEN were downregulated in ccRCC tissues compared with normal tissues. (B) The expression level of miR-19a was negatively correlated with the expression level of SMAD4 and PTEN from TCGA KIRC dataset $\left(\mathrm{R}^{2}=0.015, \mathrm{P}=0.03 ; \mathrm{R}^{2}=0.11, \mathrm{P}<0.001\right.$, respectively). (C) Western blot analysis confirmed that miR-19a inhibited the expression of SMAD4 and PTEN in 769-P cells.

level of miR-19a was negatively correlated with the expression level of both SMAD4 and PTEN (Fig. 6B, $\mathrm{R}^{2}=0.015$ and 0.11 , respectively). To validate that miR-19a can directly suppress the expression of SMAD4 and PTEN, miR-19a mimics are transfected into 769-P cells and the expression levels of SMAD4 and PTEN were detected. The results demonstrated that the expression levels of both SMAD4 and PTEN were reduced with miR-19a overexpression in 769-P cells (Fig. 6C). These data suggest that miR-19a suppresses the expression of SMAD4 and PTEN in ccRCC.

\section{Discussion}

In the present study, integrative analysis of miRNA microarray and TCGA data suggested that the expression level of miR-19a was significantly upregulated in ccRCC tissues. miR-19a, annotated with cell proliferation, was significantly correlated with $\mathrm{T}$ stage and could act as an independent prognostic marker for ccRCC. miR-19a may promote cell proliferation mainly via suppressing the expression of SMAD4/PTEN in ccRCC. These results help to provide a potential novel prognostic marker and therapeutic target for ccRCC.

A previous study demonstrated that miR-19a was aberrantly expressed in malignant glottic lesions (25) and several types of tumors, including lung (26) and gastric cancer (27). miR-19a was strongly associated with cancer invasion and metastasis, and poor prognosis of lung cancer patients (26). miR-19a could also strongly induce hepatoma cell proliferation and colony formation in vitro (28). miR-19a exerts these functions by constitutively activating the $\mathrm{NF}-\kappa \mathrm{B}$ signaling pathway (29), PI3K/Akt or JAK/STAT signaling pathway $(30,31)$. Notably, miR-19a was also reported to be upregulated in metastatic ccRCC compared with adjacent normal and primary carcinoma tissues and promoted ccRCC cell migration and invasion (32). In the present study, we further found that miR-19a not only promoted ccRCC occurence and progression, but also might 
act as a novel prognostic marker for ccRCC patients. Analysis of the results from clinical samples showed that, among T, N and $\mathrm{M}$ stages, $\mathrm{T}$ stage reflecting tumor size mostly correlated with miR-19a expression level. In vitro experiment results also revealed that miR-19a promoted ccRCC cell proliferation. These data indicate that the most important mechanism by which miR-19a leads to the poor prognosis for ccRCC may be mainly via promoting cell proliferation.

miRNAs played their roles via suppressing the expression of target mRNAs. Actually, the biological regulation between miRNAs and their targets is very complex in vivo. One miRNA may target multiple genes and target genes are tissue specific. On the basis of known target gene database, we further combined TCGA KIRC dataset and performed GSEA to find the tissue specific and key target genes of miR-19a in ccRCC. Thus, the pairs between miR-19a and its target genes found in this study would be of use in ccRCC clinic.

Furthermore, results of GO analysis revealed protein binding and the macromolecular complex were the important functions for target genes. We further performed proteinprotein interaction analysis for target genes. PPI result revealed 13 main target proteins for miR-19a in ccRCC could interact and form the complex. Among these 13 target proteins, SMAD4 and PTEN were key node proteins for the interaction among ccRCC specific target genes of miR-19a in ccRCC. That SMAD4 and PTEN acted as the ccRCC specific and key target genes of miR-19a were further verified by correlation analysis of expression levels in tissues and in vitro study. miR-19a suppressed the expression level of both SMAD4 and PTEN and promoted ccRCC cell proliferation and progression, therefore, we speculated miR-19a coordinately regulated the expression level and related signaling pathways of SMAD4 and PTEN to affect the different phases of ccRCC progression. This needs to be further experimentally investigated.

In conclusion, miR-19a is a novel potential prognostic biomarker for ccRCC, and miR-19a promoted ccRCC cell proliferation mainly by targeting PTEN and SMAD4. The present study provided a novel pathogenetic mechanism and a new treatment direction for ccRCC. To the best of our knowledge, this is the first report showing the prognostic value of miR-19a for ccRCC patients.

\section{Acknowledgements}

The present study was supported by the National Natural Science Foundation of the People's Republic of China (nos. 81372739 and 81672521).

\section{References}

1. Shim M, Song C, Park S, Choi SK, Cho YM, Kim CS and Ahn H: Prognostic significance of platelet-derived growth factor receptor- $\beta$ expression in localized clear cell renal cell carcinoma. J Cancer Res Clin Oncol 141: 2213-2220, 2015.

2. Rini BI, Campbell SC and Escudier B: Renal cell carcinoma. Lancet 373: 1119-1132, 2009.

3. Widodo, Djati MS and Rifa'i M: Role of MicroRNAs in carcinogenesis that potential for biomarker of endometrial cancer. Ann Med Surg (Lond) 7: 9-13, 2016.

4. Murugan AK, Munirajan AK and Alzahrani AS: MicroRNAs: modulators of the Ras oncogenes in oral cancer. J Cell Physiol 231: 1424-1431, 2016.

5. Yu X and $\mathrm{Li} \mathrm{Z}$ : The role of microRNAs expression in laryngeal cancer. Oncotarget 6: 23297-23305, 2015.
6. Lee SH, Jung YD, Choi YS and Lee YM: Targeting of RUNX3 by miR-130a and miR-495 cooperatively increases cell proliferation and tumor angiogenesis in gastric cancer cells. Oncotarget 6: 33269-33278, 2015.

7. Kim HS, Lee KS, Bae HJ, Eun JW, Shen Q, Park SJ, Shin WC, Yang HD, Park M, Park WS, et al: MicroRNA-31 functions as a tumor suppressor by regulating cell cycle and epithelialmesenchymal transition regulatory proteins in liver cancer. Oncotarget 6: 8089-8102, 2015.

8. Liu Y, Wang Y, Sun X, Mei C, Wang L, Li Z and Zha X: miR-449a promotes liver cancer cell apoptosis by downregulation of Calpain 6 and POU2F1. Oncotarget 7: 13491-13501, 2016.

9. Croce $\mathrm{C}$ : Introduction to the role of microRNAs in cancer diagnosis, prognosis, and treatment. Cancer J 18: 213-214, 2012.

10. Xing $\mathrm{T}$ and $\mathrm{He} \mathrm{H}$ : Epigenomics of clear cell renal cell carcinoma: Mechanisms and potential use in molecular pathology. Chin J Cancer Res 28: 80-91, 2016.

11. White NM, Bao TT, Grigull J, Youssef YM, Girgis A, Diamandis M, Fatoohi E, Metias M, Honey RJ, Stewart R, et al: miRNA profiling for clear cell renal cell carcinoma: Biomarker discovery and identification of potential controls and consequences of miRNA dysregulation. J Urol 186: 1077-1083, 2011.

12. Tang K and $\mathrm{Xu} \mathrm{H}$ : Prognostic value of meta-signature miRNAs in renal cell carcinoma: An integrated miRNA expression profiling analysis. Sci Rep 5: 10272, 2015.

13. Osanto S, Qin Y, Buermans HP, Berkers J, Lerut E, Goeman JJ and van Poppel H: Genome-wide microRNA expression analysis of clear cell renal cell carcinoma by next generation deep sequencing. PLoS One 7: e38298, 2012.

14. White NM, Khella HW, Grigull J, Adzovic S, Youssef YM, Honey RJ, Stewart R, Pace KT, Bjarnason GA, Jewett MA, et al: miRNA profiling in metastatic renal cell carcinoma reveals a tumour-suppressor effect for miR-215. Br J Cancer 105: 1741-1749, 2011.

15. Yue H, Yang BO, Yang F, Hu XL and Kong FB: Co-expression network-based analysis of hippocampal expression data associated with Alzheimer's disease using a novel algorithm. Exp Ther Med 11: 1707-1715, 2016.

16. Feitelson MA, Arzumanyan A, Kulathinal RJ, Blain SW, Holcombe RF, Mahajna J, Marino M, Martinez-Chantar ML, Nawroth R, Sanchez-Garcia I, et al: Sustained proliferation in cancer: Mechanisms and novel therapeutic targets. Semin Cancer Biol 35 (Suppl): 1707-1715, 2015.

17. Yang Q, Lin H, Wu S, Lei F, Zhu X, Song L, Hong M and Guo L: Prostate tumor overexpressed 1 (PTOV1) is a novel prognostic marker for nasopharyngeal carcinoma progression and poor survival outcomes. PLoS One 10: e0136448, 2015.

18. Yamada N, Tsujimura N, Kumazaki M, Shinohara $H$, Taniguchi K, Nakagawa Y, Naoe T and Akao Y: Colorectal cancer cell-derived microvesicles containing microRNA-1246 promote angiogenesis by activating Smad 1/5/8 signaling elicited by PML down-regulation in endothelial cells. Biochim Biophys Acta 1839: 1256-1272, 2014.

19. Chen W, Zhou S, Mao L, Zhang H, Sun D, Zhang J, Li J and Tang JH: Crosstalk between TGF- $\beta$ signaling and miRNAs in breast cancer metastasis. Tumour Biol 37: 10011-10019, 2016.

20. Preusse M, Theis FJ and Mueller NS: miTALOS v2: Analyzing tissue specific microRNA function. PLoS One 11: e0151771, 2016.

21. Zhang L, Du X, Wei S, Li D and Li Q: A comprehensive transcriptomic view on the role of SMAD4 gene by RNAimediated knockdown in porcine follicular granulosa cells. Reproduction 152: 81-89, 2016.

22. Chen J, Yan D, Wu W, Zhu J, Ye W and Shu Q: MicroRNA-130a promotes the metastasis and epithelial-mesenchymal transition of osteosarcoma by targeting PTEN. Oncol Rep 35: 3285-3292, 2016.

23. Lee CC, Yang WH, Li CH, Cheng YW, Tsai CH and Kang JJ: Ligand independent aryl hydrocarbon receptor inhibits lung cancer cell invasion by degradation of Smad4. Cancer Lett 376: 211-217, 2016.

24. Wu ZH, Tao ZH, Zhang J, Li T, Ni C, Xie J, Zhang JF and Hu XC: MiRNA-21 induces epithelial to mesenchymal transition and gemcitabine resistance via the PTEN/AKT pathway in breast cancer. Tumour Biol 37: 7245-7254, 2016.

25. Marioni G, Agostini M, Cappellesso R, Bedin C, Ottaviano G, Marchese-Ragona R, Lovato A, Cacco T, Giacomelli L, Nitti D, et al: miR-19a and SOCS-1 expression in the differential diagnosis of laryngeal (glottic) verrucous squamous cell carcinoma. J Clin Pathol 69: 415-421, 2015. 
26. Li J, Yang S, Yan W, Yang J, Qin YJ, Lin XL, Xie RY, Wang SC, Jin W, Gao F, et al: MicroRNA-19 triggers epithelial-mesenchymal transition of lung cancer cells accompanied by growth inhibition. Lab Invest 95: 1056-1070, 2015.

27. Qin S, Ai F, Ji WF, Rao W, Zhang HC and Yao WJ: miR-19a promotes cell growth and tumorigenesis through targeting SOCS1 in gastric cancer. Asian Pac J Cancer Prev 14: 835-840, 2013.

28. Baik SH, Lee J, Lee YS, Jang JY and Kim CW: ANT2 shRNA downregulates miR-19a and miR-96 through the PI3K/Akt pathway and suppresses tumor growth in hepatocellular carcinoma cells. Exp Mol Med 48: e222, 2016.

29. Yang F, Wang H, Jiang $\mathrm{Z}, \mathrm{Hu} \mathrm{A}$, Chu L, Sun $\mathrm{Y}$ and $\mathrm{Han} \mathrm{J}$ : MicroRNA-19a mediates gastric carcinoma cell proliferation through the activation of nuclear factor- $\kappa \mathrm{B}$. Mol Med Rep 12 5780-5786, 2015.
30. Li HW, Xie Y, Li F, Sun GC, Chen Z and Zeng HS: Effect of miR-19a and miR-21 on the JAK/STAT signaling pathway in the peripheral blood mononuclear cells of patients with systemic juvenile idiopathic arthritis. Exp Ther Med 11: 2531-2536, 2016.

31. Xie CY, Kong JR, Zhao CS, Xiao YC, Peng T, Liu Y and Wang WN: Molecular characterization and function of a PTEN gene from Litopenaeus vannamei after Vibrio alginolyticus challenge. Dev Comp Immunol 59: 77-88, 2016.

32. Xiao W, Gao Z, Duan Y, Yuan W and Ke Y: Downregulation of miR-19a exhibits inhibitory effects on metastatic renal cell carcinoma by targeting PIK3CA and inactivating Notch signaling in vitro. Oncol Rep 34: 739-746, 2015. 